\title{
CULTIVO E CARACTERIZAÇÃO MORFOLÓGICA DE ACESSOS DE LIPPIA ORIGANOIDES H.B.K.
}

\author{
Vanessa Franca ${ }^{1}$; Lenaldo Muniz de Oliveira ${ }^{2}$; Emily Feijó ${ }^{3}$; Romeu Leite ${ }^{4}$ \\ 1. Bolsista PIBIC/CNPq, Graduando em Agronomia, Universidade Estadual de Feira de Santana, e-mail: \\ vanessafranca2011@ hotmail.com \\ 2. Orientador, Departamento de Ciências Biológicas, Universidade Estadual de Feira de Santana, e-mail: \\ lenaldo.uefs@hotmail.com \\ 3. Doutoranda no Programa de Pós-Graduação em Recursos Genéticos Vegetais, Universidade Estadual de Feira de \\ Santana, e-mail: emillyfeijo@hotmai.com \\ 4. Bolsista PIBIC/CNPq, Graduando em Agronomia, Universidade Estadual de Feira de Santana, e-mail: \\ leiteromeu@hotmail.com
}

PALAVRAS-CHAVE: planta medicinal; variação morfológica; acessos.

\section{INTRODUÇÃO}

As plantas medicinais caracterizam-se por apresentarem um ou mais grupos de princípios ativos que lhes conferem diversas propriedades terapêuticas. Dentre as diversas famílias botânicas destaca-se a família Verbenaceae, pela elevada e variada produção de metabólitos secundários. O gênero Lippia L. possui muitas espécies de interesse medicinal, dentre elas destaca-se Lippia origanoides H.B.K. No Brasil, a espécie é popularmente conhecida como "salva-de-Marajó e "alecrim-d'Angola" (PINTO et al, 2013). O chá das folhas é usado popularmente no tratamento de diarréia, cólicas em bebês, indigestão, flatulência, febre, náuseas, complicações menstruais, azia e como anti-séptico para a boca, garganta e feridas (OLIVEIRA, 2007).

Diversos trabalhos têm comprovado a atividade antimicrobiana do óleo essencial e dos extratos da espécie contra vários microorganismos, como Candida sp. (DOS SANTOS et al., 2004; OLIVEIRA et al., 2007), Leishmania chagasi, Trypanosoma cruzi (ESCOBAR et al., 2010), T. cinnabarinus (SIVIRA et al., 2010) entre outros. Além disto, a espécie também demonstrou atividades antiviral, repelente e antioxidante.

Diversas espécies medicinais já demonstraram variações morfológicas e agronômicas em estudos de comparação de diferentes acessos. As diferenças entre os acessos são importantes, porque evidenciam a existência de variação que poderá ser utilizada em projetos de melhoramento genético, com a finalidade de selecionar indivíduos com elevada produção de metabólitos secundários e com características morfológicas e agronômicas apropriadas para o cultivo. O grupo de pesquisa de Plantas medicinais do Semiárido, liderado pelo Prof. Dr. Lenaldo Muniz de Oliveira, tem desenvolvido diversos projetos com a espécie Lippia origanoides, já havendo um volume significativo de informações da espécie, sendo de interesse a caracterização morfológica e agronômica entre os diferentes acessos já coletados, para aumentar o conhecimento cientifico sobre a espécie e, consequentemente, embasar o melhoramento genético e o cultivo da mesma, já que têm sido observadas algumas características morfológicas diferentes no Estado da Bahia.

\section{MATERIAL E MÉTODOS}

O experimento foi realizado no Horto Florestal da Universidade Estadual de Feira de Santana. A propagação foi feita por meio de mudas obtidas através de estacas dos 
acessos que formam o banco de germoplasma da espécie do Horto Florestal da UEFS. Foi realizado o plantio em sacos plásticos preto com capacidade para 1 litro, preenchidos com substrato comercial e estes permaneceram em casa de vegetação por aproximadamente 60 dias. Após esse período as mudas foram transplantadas para o local definitivo do experimento, com espaçamento de 2,0 m entre linhas e 1,0 m entre plantas. O delineamento experimental utilizado foi de blocos casualizados, com quatro repetições, constituídos de seis plantas, sendo cada acesso um tratamento.

Após 180 dias do transplante foram avaliadas as características morfológicas: cor do caule, folhas, sépalas e pétalas das flores; formato da copa; comprimento $(C)$ e largura das folhas (L), medidas com auxílio de um paquímetro $(\mathrm{cm})$ e a relação entre as duas variáveis $(\mathrm{C} / \mathrm{L})$; formato da base, limbo e ápice foliar. Ao final do experimento foram feitas medições da altura das plantas e em seguida a colheita dos acessos, sendo avaliada a massa fresca foliar (MFF) (g), utilizando a balança digital. As amostras pesadas foram levadas a estufa de circulação de ar, a $40^{\circ} \mathrm{C}$ para secagem, onde ficaram até obter peso constante, sendo, então, quantificado a massa seca foliar (MSF) (g).

Todos os resultados foram submetidos à análise de variância e suas médias comparadas pelo teste Tukey a 5\% de probabilidade de erro, através do programa estatístico SISVAR (FERREIRA, 2008).

\section{RESULTADOS E DISCUSSÃO}

A análise dos dados obtidos na caracterização morfológica dos acessos de Lippia origanoides H.B.K. evidenciou características qualitativas diferentes, permitindo a formação de dois grupos distintos. O primeiro grupo apresentou predominantemente caule marrom, folhas e nervuras das folhas verdes, assim como suas sépalas, com diferenciação na coloração das pétalas, onde alguns acessos possuem pétalas com colorações branca/roxa e outros acessos com coloração branca/roxa/amarela (Figura 1), resultando na formação de três subgrupos (Tabela 1). O segundo grupo apresentou caule marrom acinzentado, folhas e nervuras verdes, sépalas verdes e pétalas branca/amarela (Figura 2). Verificou-se ainda que o primeiro grupo apresentou o limbo com formato orbicular, ápice obtuso e base obtusa, enquanto o segundo grupo apresentou limbo de formato ovado, ápice agudo e base obtusa (Tabela 1).
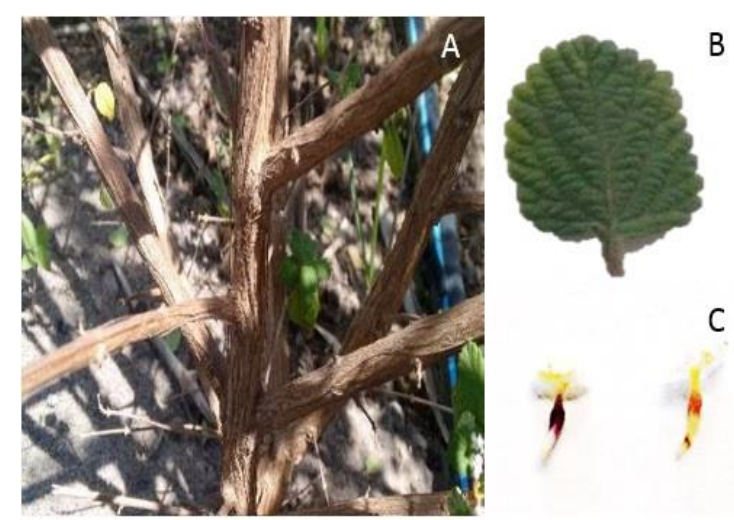

Figura 1. Lippia origanoides com sete meses em campo, 2016. Caule marrom (A), detalhes da folha (B) e coloração das pétalas (C).

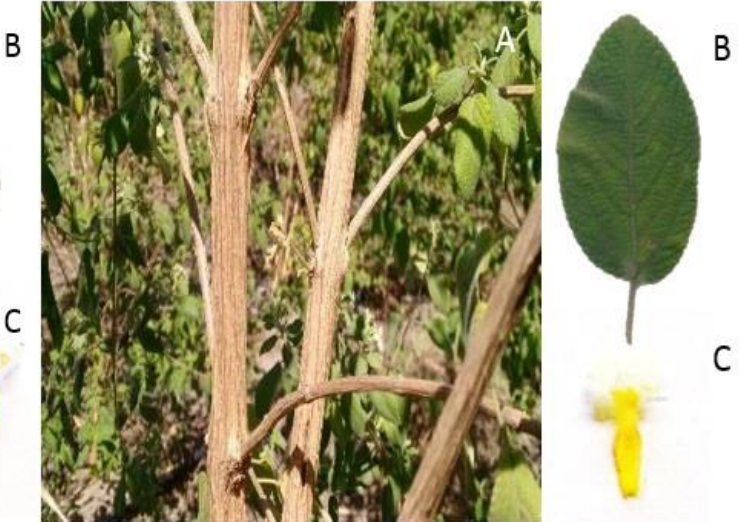

Figura 2. Lippia origanoides com sete meses em campo, 2016. Caule marrom acinzentado (A), detalhes da folha (B) e coloração das pétalas (C). 
Tabela 1. Características qualitativas dos acessos de Lippia origanoides H.B.K. do Banco de Germoplasma da Unidade Experimental Horto Florestal - UEFS.

\begin{tabular}{|c|c|c|c|c|c|c|c|c|}
\hline \multirow[t]{2}{*}{ Acessos } & \multicolumn{5}{|c|}{ Coloração } & \multicolumn{3}{|c|}{ Formato Foliar } \\
\hline & Caule & Folhas & Nervura & Sépalas & Pétalas & Limbo & Ápice & Base \\
\hline LO 01 & Marrom & Verdes & Verdes & Verdes & Bran/Rox/Ama & Orbicular & Obtuso & Obtuso \\
\hline LO 03 & Marrom & Verdes & Verdes & Verdes & Bran/Rox/Ama & Orbicular & Obtuso & Obtuso \\
\hline LO 05 & Marrom & Verdes & Verdes & Verdes & Bran/Rox & Orbicular & Obtuso & Obtuso \\
\hline LO 07 & Marrom & Verdes & Verdes & Verdes & Bran/Rox/Ama & Orbicular & Obtuso & Obtuso \\
\hline LO 08 & Marrom & Verdes & Verdes & Verdes & Bran/Rox/Ama & Orbicular & Obtuso & Obtuso \\
\hline LO 12 & Marrom Acin. & Verdes & Verdes & Verdes & Bran/Ama & Ovada & Agudo & Obtuso \\
\hline LO 13 & Marrom Acin. & Verdes & Verdes & Verdes & Bran/Ama & Ovada & Agudo & Obtuso \\
\hline LO 14 & Marrom & Verdes & Verdes & Verdes & Bran/Rox & Orbicular & Obtuso & Obtuso \\
\hline LO 16 & Marrom & Verdes & Verdes & Verdes & Bran/Rox & Orbicular & Obtuso & Obtuso \\
\hline LO 18 & Marrom Acin. & Verdes & Verdes & Verdes & Bran/Ama & Ovada & Agudo & Obtuso \\
\hline LO 19 & Marrom Acin. & Verdes & Verdes & Verdes & Bran/Ama & Ovada & Agudo & Obtuso \\
\hline LO 20 & Marrom Acin. & Verdes & Verdes & Verdes & Bran/Ama & Ovada & Agudo & Obtuso \\
\hline
\end{tabular}

*Marrom Acin. - Marrom Acinzentado; Bran/Rox/Ama - Branco, Roxo e Amarelo.

A análise estatística demonstrou diferenças entres os acessos para as características comprimento da folia, largura da folha, razão foliar e altura da planta, enquanto que para o diâmetro da copa não se detectou diferenças estatísticas (Tabela 2).

Tabela 2. Comportamento de acessos de Lippia origanoides H.B.K. do Banco de Germoplasma da UEFS para variáveis altura da planta (AP), diâmetro da copa (DC) comprimento de folha (CF), largura de folha (LF), relação comprimento/largura da folha $(\mathrm{C} / \mathrm{L})$, massa fresca de folhas (MMF), massa seca de folhas (MSF), massa fresca de caule (MFC) e massa seca de caule (MSC).

\begin{tabular}{llllllll}
\hline Acessos & AP $(\mathrm{cm})$ & DC $(\mathrm{cm})$ & CF $(\mathrm{cm})$ & LF $(\mathrm{cm})$ & C/L (cm) & $\begin{array}{l}\text { MFF } \\
\text { (g/planta) }\end{array}$ & $\begin{array}{l}\text { MSF } \\
\text { (g/planta) }\end{array}$ \\
\hline LO 01 & $169,7 \mathrm{ab}$ & $159,7 \mathrm{a}$ & $1,166 \mathrm{~b}$ & $0,866 \mathrm{~d}$ & $1,277 \mathrm{c}$ & $0,341 \mathrm{ab}$ & $0,138 \mathrm{ab}$ \\
LO 03 & $123,3 \mathrm{~b}$ & $116,1 \mathrm{a}$ & $1,078 \mathrm{~b}$ & $0,944 \mathrm{~cd}$ & $1,122 \mathrm{c}$ & $0,250 \mathrm{~b}$ & $0,100 \mathrm{~b}$ \\
LO 05 & $161,1 \mathrm{ab}$ & $149,3 \mathrm{a}$ & $1,076 \mathrm{~b}$ & $0,855 \mathrm{~d}$ & $1,255 \mathrm{c}$ & $0,415 \mathrm{ab}$ & $0,168 \mathrm{ab}$ \\
LO 07 & $142,6 \mathrm{ab}$ & $155,8 \mathrm{a}$ & $1,144 \mathrm{~b}$ & $0,844 \mathrm{~b}$ & $1,355 \mathrm{c}$ & $0,529 \mathrm{a}$ & $0,219 \mathrm{a}$ \\
LO 08 & $117,2 \mathrm{~b}$ & $125,4 \mathrm{a}$ & $1,166 \mathrm{~b}$ & $0,966 \mathrm{bcd}$ & $1,188 \mathrm{c}$ & $0,309 \mathrm{ab}$ & $0,123 \mathrm{ab}$ \\
LO 12 & $141,5 \mathrm{ab}$ & $142,9 \mathrm{a}$ & $2,233 \mathrm{a}$ & $1,321 \mathrm{a}$ & $1,666 \mathrm{~b}$ & $0,447 \mathrm{ab}$ & $0,178 \mathrm{ab}$ \\
LO 13 & $170,5 \mathrm{ab}$ & $161,8 \mathrm{a}$ & $2,172 \mathrm{a}$ & $1,266 \mathrm{a}$ & $1,683 \mathrm{~b}$ & $0,480 \mathrm{ab}$ & $0,209 \mathrm{ab}$ \\
LO 14 & $139,5 \mathrm{ab}$ & $154,8 \mathrm{a}$ & $1,186 \mathrm{~b}$ & $0,877 \mathrm{~d}$ & $1,333 \mathrm{c}$ & $0,297 \mathrm{ab}$ & $0,121 \mathrm{ab}$ \\
LO 16 & $133,7 \mathrm{~b}$ & $145,2 \mathrm{a}$ & $1,111 \mathrm{~b}$ & $0,811 \mathrm{~d}$ & $1,333 \mathrm{a}$ & $0,471 \mathrm{ab}$ & $0,195 \mathrm{ab}$ \\
LO 18 & $213,7 \mathrm{a}$ & $163,9 \mathrm{a}$ & $2,466 \mathrm{a}$ & $1,158 \mathrm{abc}$ & $1,932 \mathrm{a}$ & $0,437 \mathrm{ab}$ & $0,176 \mathrm{ab}$ \\
LO 19 & $151,1 \mathrm{ab}$ & $126,7 \mathrm{a}$ & $2,288 \mathrm{a}$ & $1,218 \mathrm{ab}$ & $1,655 \mathrm{~b}$ & $0,329 \mathrm{ab}$ & $0,140 \mathrm{ab}$ \\
LO 20 & $149,7 \mathrm{ab}$ & $140,5 \mathrm{a}$ & $2,188 \mathrm{a}$ & $1,210 \mathrm{abc}$ & $1,777 \mathrm{ab}$ & $0471 \mathrm{ab}$ & $0,187 \mathrm{ab}$ \\
\hline CV $(\%)$ & 18,53 & 15,46 & 9,82 & 9,84 & 6,25 & 25,48 & 25,99 \\
\hline
\end{tabular}

*Valores com letras iguais, nas colunas, não diferem entre si pelo teste de Tukey $(p \leq 0,10)$.

As plantas variaram entre 117,2 a 213,7 centímetros de altura, sendo o acesso LO 08 de altura mínima e o LO 18 altura máxima. O comprimento foliar variou entre 1,076 a 2,466 cm, tendo os acessos LO12, LO13, LO18, LO19, LO20 maiores dimensões. Os acessos LO12 e LO13 apresentaram maior largura foliar. Na relação comprimento/largura de folha o acesso LO18 se sobressaiu, com média de 1,932. Verificou-se maior destaque para os acessos LO12, LO13, LO18, LO19 e LO20, definidos anteriormente como grupo dois. 
As diferenças observadas nas características morfológicas entre os acessos de $L$. origanoides podem ser atribuídas a variações genéticas entre os acessos, uma vez que, mesmo coletados em municípios diferentes, foram cultivados e avaliados em um mesmo local e nas mesmas condições experimentais, resultando em variações fenotípicas. Diferenças genéticas também foram observadas por Camêlo et al. (2011) ao avaliar características morfológicas e agronômicas em acessos de Lippia alba (Mill) N.E.Br., também conhecida como erva-cidreira-brasileira, onde foram avaliadas as variáveis comprimento de ramo, diâmetro de copa, cor de caule, folhas, pétalas, comprimento e largura de folha e relação comprimento/largura de folha.

De modo semelhante, verificou-se diferenças significativas para as características agronômicas massa fresca e seca de folhas entre os acessos avaliados (Tabela 02). $\mathrm{O}$ acesso LO07 expressou o melhor resultado, com massa fresca e seca $(0,529$ e 0,219 $\mathrm{g} /$ planta, respectivamente). Ressalta-se que a massa seca de folhas é um parâmetro de grande importância para a espécie, cujo produto principal é o óleo essencial, produzido em maior quantidade nas folhas.

A partir das variações morfológicas e agronômicas obtidas verifica-se que existe variação genética entre os 12 genótipos avaliados e que os acessos LO07, LO12, LO13, LO18, LO19 e LO20 apresentam potencial para uso em programas de melhoramento, que envolvam hibridação com vistas à exploração da heterose, visando à obtenção de bons materiais para produção de biomassa foliar e, consequentemente, maior rendimento de óleo essencial.

\section{CONCLUSÕES}

Existe variabilidade morfológica e agronômica entre acessos de L. origanoides H.B.K. que permite a seleção e/ou hibridação entre os acessos, com vistas ao melhoramento genético da espécie.

\section{REFERÊNCIAS}

CAMELO, L. C. A. et al. Caracterização morfológica e agronômica de acessos de ervacidreira-brasileira [Lippia alba (Mill.) N. E. Br.]. Scientia Plena, Aracajú, v. 7, p. 1-8, 2011.

DOS SANTOS, F.J.B., et al. Composition and biological activity of essential oils from Lippia origanoides H.B.K. J. Essent. Oil Res., v.16, p.504-506, 2004.

ESCOBAR, P. et al. Chemical composition and antiprotozoal activities of Colombian Lippia spp essential oils and their major components. Memórias do Instituto Oswaldo Cruz, v. 105, p.184-190, 2010.

OLIVEIRA, D. R. et al. Chemical and antimicrobial analyses of essential oil of Lippia origanoides H.B.K. Food Chemistry, v. 101, n. 1, p. 236-240, 2007.

PINTO, C.P. et al. Antimicrobial Activity of Lippia Species from the Brazilian Semiarid Region Traditionally Used as Antiseptic and Anti-Infective. Agents EvidenceBased Complementary and Alternative Medicine, 2013.

SALIMENA, F.R.G. Novos sinônimos e tipificação em Lippia sect. Rhodolippia (Verbenaceae). Darwiniana, v.40, p.121-125, 2002.

SIVIRA, A. et al. Toxicity of ethanolic extracts from Lippia origanoides and Gliricidia sepium to Tetranychus cinnabarinus (Boisduval) (Acari: Tetranychidae). Neotropical Entomology, v. 40, n.3, p.375-379, 2011. 